

\title{
Targeting $T$ cell activation in immuno-oncology
}

\author{
S.D. Saibil $\mathrm{MD} \mathrm{PhD}^{* \dagger}$ and P.S. Ohashi $\mathrm{PhD}^{* \neq}$
}

\begin{abstract}
The years since 2009 have seen tremendous progress in unlocking the curative potential of the immune system for the treatment of cancer. Much of that revolution in immuno-oncology has been fueled by the clinical success of immune checkpoint inhibitors, particularly those targeting the PD-1 axis. Unfortunately, many patients still fail to benefit from checkpoint blockade or other immunotherapies. An inability to fully activate antitumour T cells contributes in part to the failure of those therapies. Here, we review the basic biology of T cell activation, with particular emphasis on the essential role of the dendritic cell and the innate immune system in T cell activation. The current understanding of the multiple factors that govern $\mathrm{T}$ cell activation and how they impinge on tumour immunotherapy are also discussed. Lastly, treatment strategies to potentially overcome barriers to $\mathrm{T}$ cell activation and to enhance the efficacy of immunotherapy are addressed.
\end{abstract}

Key Words Immuno-oncology, T cell activation, immune checkpoint inhibitors

Curr Oncol. 2020 April:27(S2)98-105

www.current-oncology.com

\section{INTRODUCTION}

Immune checkpoint inhibitors (ICIs) - antibodies targeting either CTLA-4 (CD152), PD-1 (CD279), or PD-L1 (CD274)have revolutionized immuno-oncology. Patients with tumours of varying histologies have experienced impressive survival advantages after treatment using ICI agents compared with treatment using the previous standard-ofcare agents $^{1}$. Despite that success, the reality is that most patients treated with ICIs will not achieve a significant clinical response.

Although frustrating from a clinical perspective, the lack of response to ICI monotherapy in most patients is perhaps not surprising in the context of T cell biology. It is now appreciated that myriad intricate regulatory mechanisms control $\mathrm{T}$ cell functioning, particularly in the context of antitumour immunity ${ }^{2}$. A key factor governing the successful activation of the T cell arm of the adaptive immune system is the requirement for the coordinated activation of the innate immune system, which consists of cells such as macrophages, innate lymphoid cells, natural killer cells, and dendritic cells (DCs). In particular, $\mathrm{T}$ cell activation is highly dependent on the functioning of DCs, which present antigens to $T$ cells and bridge the activation of the innate immune system to the adaptive immune system ${ }^{3}$. Here, we review the regulation of $\mathrm{T}$ cell activation within the context of tumour immunotherapy.
We also highlight opportunities and therapeutic strategies that aim to increase $\mathrm{T}$ cell activation, thereby enhancing the efficacy of ICIs and other immunotherapies.

\section{MECHANISMS}

\section{T Cell Tolerance: A Fine Balance}

The fundamental challenge of $\mathrm{T}$ cell immunity is for the system to respond to a universe of pathogens while limiting autoreactivity. The immune system must be able to develop a repertoire of $\mathrm{T}$ cells that encode antigen receptor specificities sufficiently diverse to recognize the wide range of antigens potentially present in pathogens. Conversely, the $\mathrm{T}$ cell response must be constrained from reacting to antigens present in self-proteins. For T lymphocytes, this balancing act begins in thymus, during a process called "central tolerance" or "thymic selection"4.

In the thymus, immature $\mathrm{T}$ cells randomly rearrange the variable, diversity, and joining segments of their T cell receptor (TCR) genes to generate a vast array of TCR specificities $^{5}$. For a $\mathrm{T}$ cell to mature and leave the thymus, the variable, diversity, and joining rearrangements must result in a TCR that is capable of recognizing peptide antigens presented in the context of the individual's own major histocompatibility complex [MHC (human leucocyte antigen)] molecules. This process is called "positive selection," because it selects T cells expressing receptors appropriate 
to survival and differentiation. Alternatively, T cells expressing TCRs that react with high affinity to the self-peptide MHC complexes present in the thymus are instructed, in a process called "negative selection," to undergo cell death. Thus, negative selection is a mechanism to prevent $\mathrm{T}$ cells that are strongly autoreactive from leaving the thymus and potentially causing autoimmunity.

Unfortunately, negative selection is not perfect, and mature self-reactive cells can be found outside the thymus, even in individuals who do not demonstrate any signs of autoimmune disease ${ }^{6}$. Accordingly, other regulatory mechanisms are required to prevent the induction of autoimmunity by the self-reactive clones that have avoided thymic deletion. Other cells of the immune system-such as specialized $\mathrm{T}$ regulatory cells (Tregs), and cells of the innate immune system such as DCs-play pivotal roles in regulating activation or induction of immune tolerance for clones that have left the thymus.

\section{T Cell Activation}

Stimulation of a TCR in a mature T cell with a cognate antigen-MHC complex can lead to opposing outcomes. Signals from the TCR can induce T cell activation when delivered with the appropriate additional costimulatory signals. Conversely, signals from the TCR can induce T cell tolerance by causing deletion of the T cells or induction of cells that display a hyporesponsive state called "anergy," the hallmarks of which are lack of proliferation and low production of interleukin 2 (IL-2) ${ }^{7,8}$. The functional state of DCs has emerged as a key determinant of the decision between $\mathrm{T}$ cell activation and tolerance. Immature or non-activated DCs have low levels of MHC complexes and costimulatory ligands. Upon maturation, DCs dramatically increase the expression of MHC peptide complexes and of the costimulatory ligands in addition to T cell-stimulating cytokines. Thus, the current model of $\mathrm{T}$ cell activation is that immature DCs are tolerogenic and induce T cell tolerance through deletion or anergy and that mature DCs are activating and induce a robust immune response against the antigens they present ${ }^{9,10}$ (Figure 1).

The pattern recognition receptor (PRR) family of receptors plays a central role in mediating the activation of DCs. The PRR family members are expressed on immature DCs and induce DC maturation and activation upon binding of their ligand ${ }^{3}$. The toll-like receptors (TLRs) constitute one of the best-characterized groups of PRR family members. The TLRs recognize unique bacteria or viral molecules, called pathogen-associated molecular patterns (PAMPs). More recently, however, it has been discovered that, in addition to PAMPs, certain endogenous molecules can activate PAMPs. Those molecules, called damage-associated molecular patterns (DAMPs), can be molecules typically located in the nucleus (such as DNA or histones), cytosolic molecules (such as ATP), and molecules derived from the extracellular matrix (such as biglycan) ${ }^{11}$. The DAMPs can be recognized by a variety of PRR family members, including some of the TLRs and other receptors such as MDA5 and the cGAS-STING pathway, resulting in the induction of local inflammation and DC maturation. The exposure of DCs to PAMPs and DAMPs therefore influences the activation status of the DCs and, subsequently, $\mathrm{T}$ cell activation or tolerance.

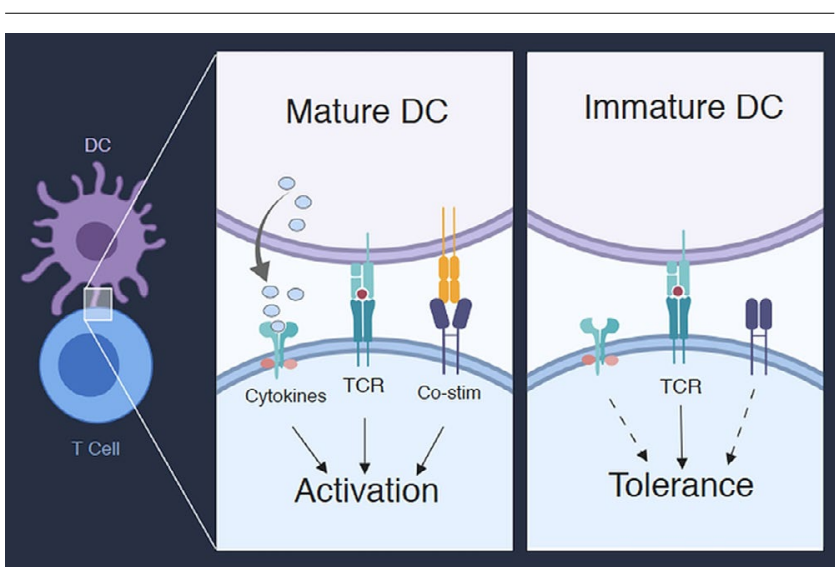

FIGURE 1 The maturation status of dendritic cells (DCs) determines T cell activation or tolerance. Mature DCs provide T cells with signals through the T cell receptor (TCR) and through co-stimulatory receptors (co-stim) and cytokines, resulting in T cell activation. Immature DCs provide signals only through the TCR, which results in T cell tolerance. Maturation of DCs requires signals from the pattern recognition family of receptors.

Upon activation, DCs provide multiple costimulatory signals to T cells. Those signals can be delivered by ligation of specialized costimulatory receptors expressed on the $\mathrm{T}$ cell or by provision of inflammatory cytokines such as interleukin 12, or both ${ }^{12}$. Multiple receptors expressed on $\mathrm{T}$ cells have been demonstrated to provide costimulatory signals for T cell activation ${ }^{13}$. Most of those receptors belong either to the immunoglobulin superfamily (such as CD28) or the tumour necrosis factor receptor superfamily [such as 4-1BB (CD137 or TNFRSF9) and OX40 (TNFSF4)]. All of those costimulatory receptors contain intracellular signalling domains that synergize with signals from the TCR and that induce $\mathrm{T}$ cell proliferation, effector function, and survival. Figure 2 outlines a selection of costimulatory receptors expressed on $\mathrm{T}$ cells and their known cognate ligands expressed on DCs. The precise role that the individual costimulatory receptor and ligand pairs play in $\mathrm{T}$ cell activation and differentiation is still not fully elucidated and is currently an area of active investigation.

\section{THERAPEUTIC OPPORTUNITIES}

\section{Targeting DC and T Cell Activation Pathways \\ Targeting PRR Family Receptors}

In the context of tumour immunotherapy, there has been interest in using PAMPs and DAMPs to increase DC activation and antitumour immunity. Unlike pathogens, tumours do not express traditional viral or bacterial PAMPs to induce DC activation. The most direct strategy to overcome the lack of conventional PAMPs is through the direct introduction of those molecules. The concept was first demonstrated clinically near the start of the 1980s, with the local immunologic response generated against bladder cancer by the intravesicular instillation of the attenuated bacteria bacillus Calmette-Guérin ${ }^{14}$. More recent reports from preclinical models have suggested that intratumoural treatment with TLR ligands can boost antitumour immunity both locally and at tumour sites distant to the injection, particularly 


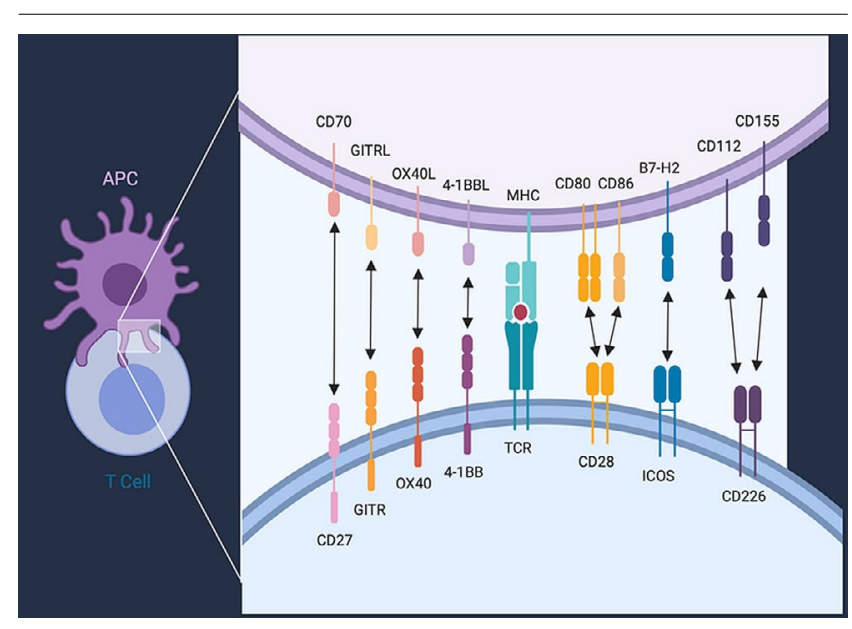

FIGURE 2 Selection of co-stimulatory receptors and their ligands. Members of the immunoglobulin superfamily co-stimulatory receptors and their ligands depicted are CD28, inducible $\mathrm{T}$ cell co-stimulator (ICOS, CD278), and CD226. Members of the tumour necrosis factor receptor superfamily co-stimulatory receptors and their ligands depicted are CD27 (TNFRSF7), GITR (TNFRSF18), OX40 (CD134/TNFSF4), and 4-1BB (CD137/TNFRSF9). Abbreviations or other names for the ligands are B7-1 (CD80), B7-2 (CD86), ICOS ligand (ICOSL/B7-H2/CD275), OX40 ligand (OX40L/CD252), 4-1BB ligand (CD137L), GITR ligand (GITRL/TL6), and CD70 (TNFSF7). APC = antigen presenting cell.

when combined with other immune-stimulating treatments such as checkpoint blockade. In mouse models, such intratumoural injections can activate DCs in the tumour microenvironment, which, upon activation, migrate to regional lymph nodes to prime the antitumour $\mathrm{T}$ cell response. Hence, the responses generated by the injections can result in tumour regression at sites distant to the injected lesions ${ }^{15,16}$. Currently, multiple clinical trials investigating the clinical efficacy of intratumoural injections of TLR agonists, mainly in combination with other immuno-oncology agents, are underway ${ }^{17}$.

An alternative strategy to boost DC activation has been to produce synthetic ligands for the PAMPs. Of all the receptors studied so far, the cGAS-STING signalling pathway has appeared particularly attractive for this approach. A cytosolic PRR, cGAS is activated downstream of cytosolic DNA. Once activated, cGAS activates STING, which results in the activation of DCs and the expression of pro-inflammatory cytokines such as type I interferons ${ }^{18}$. Recent work in mice has suggested that activation of STING within the DCs in the tumour microenvironment is crucial for the production of type I interferons and generation of an antitumour $\mathrm{T}$ cell response $^{19}$. Importantly, recently identified STING agonist compounds that can be administered systemically instead of by intratumoural injection were found to mediate impressive STING-dependent tumour regression in a preclinical model $^{20}$. These agents hold the promise of a therapeutic modality that can induce DC maturation even in tumours not amenable to intratumoural treatments.

\section{Vaccine Approaches}

Vaccination is another therapeutic strategy to induce DC maturation and T cell immunity. Historically, tumour vaccines have not demonstrated significant clinical efficacy, particularly in the treatment of metastatic disease ${ }^{21}$. However, the increased understanding of DC and T cell biology has led to new vaccine designs with better selection of the tumour target antigens and the vaccine adjuvants that trigger PAMPs, ensuring DC maturation ${ }^{22}$. There has been great interest in exploring the use of tumour "neoantigens" as vaccine targets.

Tumour neoantigens arise because of nonsynonymous mutations that result in amino acid substitutions and the generation of novel peptides that can potentially be recognized by the immune system. The clinical excitement about neoantigens arises from the observation that tumours with a high tumour mutational burden-and hence more neoantigens-show increased responsiveness to ICI therapy ${ }^{23,24}$. A recent study demonstrated a correlation of increased tumour mutational burden with patient survival after ICI therapy in multiple tumour types ${ }^{25}$. However, it is still unclear whether the neoantigens are themselves the direct targets of antitumour immunity, as has been suggested by some findings in preclinical models of ICI therapy ${ }^{26}$, or if they are a surrogate marker for tumours with genetic instability, for which high tumour mutational burden might trigger the innate immune system by neoantigen-independent mechanisms. Despite the uncertainty, personalized vaccine strategies targeting patient-specific neoantigens have shown some promise in early-phase trials ${ }^{27,28}$. Furthermore, the improved vaccine platforms used in the relevant studies hold a promise to induce DC and T cell activation and to bolster antitumour immunity to multiple different target antigens.

\section{Oncolytic Viruses}

Other therapeutic agents that potentially act by inducing DC and $T$ cell activation include modified viruses. These engineered ("oncolytic") viruses were originally developed as agents to induce direct lysis of tumour cells. However, further research has indicated that a major mechanism of the antitumour effect of the viruses is to stimulate the antitumour immune response ${ }^{29}$. Oncolytic viruses stimulate the antitumour immune response by multiple mechanisms. Given that they are viruses, they encode viral PAMPs that can potentially activate DCs directly. They also infect tumour cells and result in cell lysis, thereby promoting the release of DAMPs and tumour antigens. Oncolytic viruses can also be engineered to express inflammatory cytokines and chemokines. For example, talimogene laherparepvec (T-VEC) is a modified herpes simplex virus type 1 , designed to selectively replicate in and lyse tumour cells. The virus also encodes the gene for encoding human granulocyte-macrophage colony-stimulating factor to attract and activate DCs. Talimogene laherparepvec is the first-in-class oncolytic virus to be approved for clinical use by the U.S. Food and Drug Administration. That approval was granted based on a demonstrated therapeutic benefit of the injections in patients with unresectable stages IIIB-IV melanoma ${ }^{30}$. Trials with other oncolytic viruses are ongoing, and those agents might prove to be useful tools to induce an antitumour $\mathrm{T}$ cell response ${ }^{29}$.

\section{Cytokines}

In many cases, the immune response has already been triggered in patients through natural immune surveillance, 
leading to detectable infiltration of CD8+ T cells into the tumour. Early immune therapies, such as provision of cytokines, acted to improve those natural tumour-specific surveillance mechanisms. Historically, high-dose IL-2 and interferon alpha have been used in the treatment of renal cell carcinoma and melanoma, with limited benefit ${ }^{31}$. More recently, multiple other cytokines that improve $\mathrm{T}$ cell and natural killer cell survival and function-such as IL-12, IL-15, and IL-21-have been tested in early clinical trials ${ }^{32}$. The toxicity of systemic administration of those cytokines has been a major issue in most of their trials. In the attempt to minimize toxicity but maintain efficacy, modified versions of the cytokines are being developed. For example, bempegaldesleukin, a polyethylene glycol-conjugated recombinant IL-2, has demonstrated antitumour activity but acceptable toxicity in animal models ${ }^{33}$ and is currently being tested in the clinic in combination with ICIs.

\section{Costimulatory Agonists: Investigating New Targets}

Given the importance of costimulatory signals for $\mathrm{T}$ cell activation, agonist antibodies targeting receptors such as 4-1BB and OX40 have been investigated as an additional strategy to enhance $T$ cell activation. In preclinical models, agonistic antibodies targeting many of these costimulatory receptors have demonstrated the ability to increase T cell activation and promote tumour eradication, which has led to many being tested in clinical trials ${ }^{34}$. A current challenge is to fully understand the context-dependent role the each of these costimulatory receptors plays in the $\mathrm{T}$ cell response against various tumour types and to discover which patient populations would potentially benefit from treatment with a given agonistic antibody. Toxicity has also been a problem with the clinical development of some of these agents. For instance, hepatic toxicity was a major concern in the development of the agonistic anti-4-1BB antibody urelumab $^{35}$. Thus, further studies are required to define and optimize the clinical utility of those agents as tools to promote $\mathrm{T}$ cell activation and antitumour immunity.

\section{Strategies of Adoptive T Cell Therapy}

Instead of attempting to promote $\mathrm{T}$ cell activation in vivo, another therapeutic strategy is the transfer of ex vivo activated, tumour-specific T cells in an adoptive cell therapy approach. Early trials using polyclonal T cells expanded from tumour-infiltrating lymphocytes demonstrated impressive response rates in patients with melanoma before the era of ICIs ${ }^{36}$. More recent iterations of those cellular therapies include the use of modified T cells that have been genetically engineered to recognize tumour cells. Such technologies include chimeric antigen receptor $\mathrm{T}$ cells, which hold the promise to revolutionize the treatment of hematologic malignancies ${ }^{37}$. Tumour regression has also been achieved in solid tumours using $\mathrm{T}$ cells that are engineered to express a high-affinity TCR that recognizes a peptide derived from a protein whose expression is enriched for certain tumours, such as the cancer/testis antigen New York esophageal squamous cell carcinoma $1^{38,39}$. These cellular treatment protocols also offer the exciting opportunity to use genetic or pharmacologic means to further enhance T cell activation ex vivo before the T cells are infused.

\section{Negative Regulation of T Cells}

\section{Checkpoint Inhibitors: Beyond PD-1 and PD-L1}

Counteracting the activity of the costimulatory receptors are multiple negative regulatory receptors (such as PD-1) that are expressed by T cells. Many of the negative regulatory receptors, also called immune checkpoints or co-inhibitory receptors, are expressed on T cells only after activation. The co-inhibitory receptors (akin to the costimulatory receptors) also belong mainly to either the immunoglobulin superfamily or the tumour necrosis factor receptor superfamily and have at least 1 identified ligand ${ }^{13}$. Figure 3 depicts a selection of those receptors and their ligands.

Ligation of the co-inhibitory receptors results in the intracellular activation of signalling molecules such as phosphatases that oppose $\mathrm{T}$ cell activation in part by targeting signals from costimulatory receptors. For example, ligation of PD-1 on activated T cells has been demonstrated to antagonize activating signals downstream of costimulatory receptors, particularly $\mathrm{CD} 28^{40}$. Interestingly, although co-inhibitory receptors block signals from costimulatory receptors, they are not primarily associated with induction of T cell anergy or deletion. In the context of persistent antigen and inflammation, signals downstream of the inhibitory receptors have been found to limit $\mathrm{T}$ cell activation by inducing a hypofunctional state called T cell exhaustion (Tex).

Tex is a distinct cellular state that can be defined by a unique metabolic, epigenetic, and transcriptional signature and that is clearly distinct from both the activated and anergic states ${ }^{41,42}$. The functional characteristics of Tex are best described in the CD8+ T cell compartment, because they were first defined in the noncytopathic lymphocytic choriomeningitis virus clone 13 chronic infection model. In mice with a chronic version of that infection, virus-specific

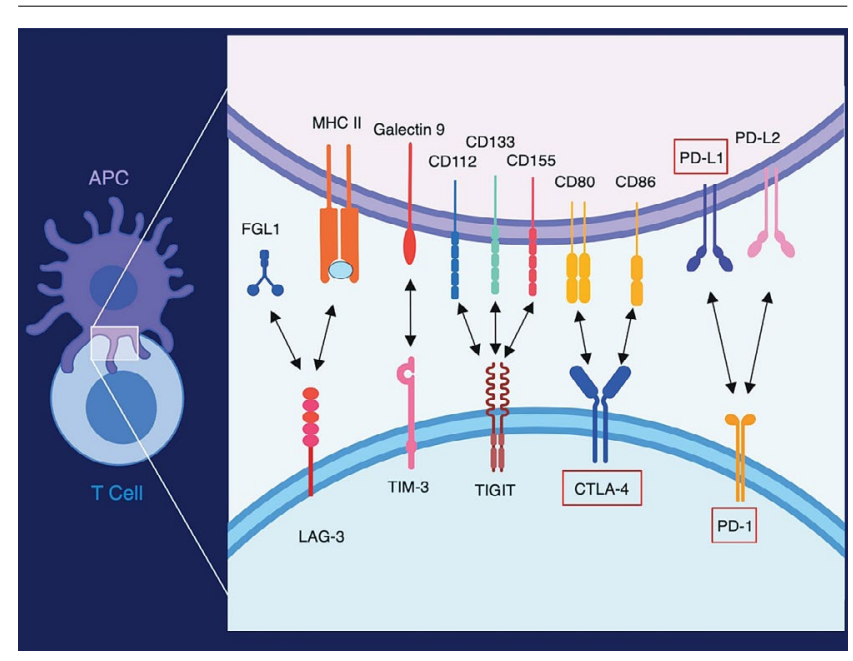

FIGURE 3 Selection of co-inhibitory receptors and their ligands. Co-inhibitory molecular interactions include LAG-3 (CD223), TIM-3 (HAVCR2/CD366), TIGIT (VSTM3), CTLA-4 (CD152), and PD-1 (CD279). Abbreviations or other names for the ligands are FGL1, B7-1 (CD80), B7-2 (CD86), PD-L1 (B7-H1/CD274), and PD-L2 (B7-DC/CD273). Red boxes indicate molecules for which antibodies that block the interaction with the molecule's target ligand are approved for clinical use. APC = antigen presenting cell. 
CD8+ T cells that accumulated over the course of infection demonstrated reduced functionality ${ }^{43,44}$. Those Tex showed a hierarchal loss of effector function that depended on the persistence of cognate antigen and the accumulation of expression of negative regulatory receptors. That sequence of functional loss, which starts with reduced production of IL-2 and then proceeds to defects in tumour necrosis factor $\alpha$ production and finally to interferon $\gamma$ production and cytotoxicity, is a hallmark feature of the development of Tex in both chronic infections and cancer. The Tex are believed to lose function as a mechanism of peripheral tolerance that prevents immunopathology in the face of persistent antigen. Conversely, the functional loss in Tex is also likely a barrier to productive antitumour immunity.

The discovery that the negative regulatory receptors provided "druggable" targets to modulate T cell function was a major advance in the field of immuno-oncology. Seminal work using a tumour model and the noncytopathic lymphocytic choriomeningitis virus infection model demonstrated that using blocking antibodies to one of the immune checkpoint receptors, PD-1, or its ligand, PD-L1, to prevent signals to CD8+ T cells could improve T cell effector function and promote tumour or viral clearance ${ }^{45,46}$. However, a major challenge in the immuno-oncology field is to fully understand the function of all the immune checkpoint receptors. As discussed earlier, in addition to the PD-1/-L1 signalling axis, T cells express multiple other negative regulatory receptors under conditions of activation and exhaustion (Figure 3). Therapeutic antibodies against many of the negative regulatory receptors-for example, LAG-3 and TIGIT-have already been developed and are currently in clinical trials.

Unfortunately, a mechanistic understanding of the complex biology of each of the inhibitory receptors is still lacking. For example, although anti-LAG-3 antibodies are currently in phase II/III clinical trials, a recent report has just identified a novel ligand for LAG-3, FGL-1, revealing a more complex biology to be understood ${ }^{47}$. Accordingly, designing clinical trials based on rational combinations of the antibodies has proved to be challenging because the precise role for each of the negative receptors in modulating $\mathrm{T}$ cell function (or other cells) remains to be elucidated. Importantly, combining agents that target different inhibitory receptors remains a practical approach, with the most striking example being the synergy demonstrated between anti-PD-1 and anti-CTLA-4 agents in the treatment of various tumours ${ }^{48}$. Thus, further work is required to determine the optimal combinations for the therapeutic agents already in various phases of clinical trial.

\section{Tregs}

In addition to co-inhibitory receptors, multiple regulatory cell populations oppose $\mathrm{T}$ cell activation. One of the best-characterized of the regulatory cell populations is the CD4+ T cell subset that express the lineage-defining transcription factor FOXP3 in addition to constitutively high levels of CD25 (IL-2 receptor $\alpha$ chain) and CTLA-4. Those cells - the Tregs - were first discovered by Sakaguchi and colleagues $^{49}$ to have an important role in promoting peripheral tolerance and preventing autoimmunity. Sakaguchi et al. demonstrated that mice depleted of CD4+CD25+
$\mathrm{T}$ cells developed a multi-system autoimmune disease. Subsequently, it was discovered that FOXP3 was the lineagedefining transcription factor governing the development of Tregs and that mice and humans with mutations in the gene encoding FOXP3 also develop a profound autoimmunity ${ }^{50-52}$. Mechanistically, Tregs appear to have multiple ways to prevent autoimmunity. Tregs can suppress $\mathrm{T}$ cell activation by inhibiting the activation of DCs. Tregs can also directly suppress $\mathrm{T}$ cell activation using both poorly defined cell contact-dependent mechanisms and contact-independent mechanisms, such as the production of immunosuppressive cytokines such as transforming growth factor $\beta^{53}$.

Within the context of immunotherapy and tumour biology, Tregs have also been found to play an important role. Depletion of Tregs from tumours can lead to enhanced tumour clearance in murine tumour models ${ }^{54,55}$. In patients, a low ratio of CD8+ T cell effectors to Tregs in the tumour microenvironment has been linked with poor prognosis in multiple tumour types ${ }^{56}$. Collectively, work by many researchers has demonstrated a critical role of CD4+ FOXP3+ Tregs in influencing the function of $\mathrm{T}$ cells in the context of both autoimmunity and cancer.

Depletion of negative regulatory cells is an attractive therapeutic strategy to increase T cell activity. It has been proposed that depletion of Tregs in the tumour microenvironment through antibody-dependent cellular cytotoxicity forms part of the antitumour effect of the anti-CTLA- 4 antibody ipilimumab ${ }^{57,58}$, although that mechanism of action remains a matter of debate ${ }^{59}$. An important aim of immunotherapy research is to discover agents that can specifically deplete Tregs in the tumour microenvironment and thereby augment the antitumour immune response. The challenge with the development of such agents is avoid systemically depleting Tregs and inducing autoimmunity. Targeting the chemokine receptor CCR8 might have such potential, because two recent studies suggested that CCR8 is specifically expressed on Tregs in the tumour microenvironment ${ }^{60,61}$. Studies have also shown that an anti-CCR8 antibody can deplete Tregs in the tumour microenvironment and improve the immunoresponse ${ }^{62}$. Targeting other molecules, including CD25 and CCR4, to deplete Tregs in the tumour microenvironment is also being actively pursued ${ }^{55,63}$.

\section{Innate Immune Cells}

In addition to Tregs, other cells found in the tumour microenvironment can modulate $\mathrm{T}$ cell activation and function. Cells of the myeloid lineage, such as macrophages and myeloid-derived suppressor cells (MDSCs), have important roles in regulating $\mathrm{T}$ cell activation within the tumour microenvironment ${ }^{64,65}$. One mechanism that MDSCs and tumour-associated macrophages (TAMs) both use to suppress $\mathrm{T}$ cell activation is production of the immuno-regulatory enzymes IDO and arginase 1 . Those enzymes degrade and starve $T$ cells of, respectively, the amino acids tryptophan and arginine, which are required to sustain T cell activation ${ }^{66}$. In addition, IDO results in the production of kynurenine and its metabolites, molecules with emerging immunomodulatory functions.

In addition to TAMs and MDSCs, novel subsets of cells with regulatory properties continue to be described. In 
patients with high-grade serous ovarian cancer and nonsmall-cell lung cancer, recent studies have described a population of innate lymphoid cells that demonstrated an ability to suppress T cells ex vivo ${ }^{67,68}$. Further work is required to better define the role of all of those suppressive cell types in tumour biology and in peripheral tolerance. It is clear that multiple immune cell populations of the innate immune system can regulate the immune response, particularly in the context of the tumour microenvironment.

As in Treg-depleting therapeutics, reagents are being developed to deplete TAMs and MDSCs from the tumour microenvironment ${ }^{69,70}$. For example, an agonistic antibody targeting TRAIL-R2 was recently shown to be able to deplete MDSCs from tumours in about half the patients treated on a phase I trial ${ }^{71}$. Additionally, as opposed to depleting TAMs and MDSCs from the tumour microenvironment, reagents to inhibit the enzymatic activity of arginase 1 and IDO are also being evaluated ${ }^{66}$. Great clinical excitement attended the early-phase efficacy data for a combination of an anti-PD-1 agent and the IDO inhibitor epacadostat (aka INCB24360), particularly for the treatment of melanoma. Unfortunately, the phase III trial ECHO-301/KEYNOTE-252 (see NCT02752075 at https://ClinicalTrials.gov/) was halted because of a lack of added clinical benefit for the combination compared with anti-PD-1 alone. Further trials are needed to test whether other IDO inhibitors that potentially have more potent enzymatic inhibition will result in clinical benefit or whether a biomarker that will predict clinical response to IDO inhibition can be defined. However, targeting negative regulatory cells or their effector mechanisms remains an attractive therapeutic avenue.

\section{Emerging Considerations for T Cell Activation: Microbiome and Metabolism}

Mammals are colonized by billions of commensal bacteria, particularly within the gastrointestinal tract. It was first noted in preclinical models that mice with different intestinal microbiota displayed differing responses to anti-PD-1 therapy when implanted with the same tumour ${ }^{72}$. That finding was then extended to humans, because patients with melanoma who responded to anti-PD-1 therapy were discovered to have a more diverse gut microbiome than did patients who did not respond to treatment ${ }^{73}$. Similarly, in a retrospective analysis, depletion of the microbiome with antibiotics was found to decrease the efficacy of ICI therapy in multiple tumour histologies ${ }^{74}$. Collectively, those data indicate an important relationship between the intestinal bacteria and the immune system. However, the precise mechanisms through which the commensal bacteria affect ICI therapy efficacy has not been determined.

Interestingly, commensal bacterial have been implicated in regulating the circulating level of multiple metabolites, particularly short-chain fatty acids such as acetate, butyrate, and propionate ${ }^{75}$. In mouse models, all of those molecules have been found to affect T cell activa$\operatorname{tion}^{76,77}$. Further studies are required to investigate if those microbial-regulated metabolites do indeed contribute to the influence of the microbiome on ICI therapy. However, it seems likely that future adjuncts to ICI will be aimed at altering the microbiome itself or its metabolic products.

\section{SUMMARY}

The regulation of $T$ cell activation is complex, with multiple levels of control, many of which involve the activation of the innate immune system-and specifically DCs. Many of the regulatory mechanisms evolved to prevent the activation of self-reactive $T$ cells and the development of autoimmunity. However, in immunotherapy, those same mechanisms limit $\mathrm{T}$ cell activation and the curative potential of immuno-oncology treatment strategies. Moreover, many of the mechanisms regulating $\mathrm{T}$ cell activation are dynamic, and blocking one might result only in the induction of another. Accordingly, combination therapies that target multiple aspects of T cell activation are the future of immunotherapy. The challenge will be to devise treatment strategies that can lead to T cell activation without causing intolerable systemic autoimmunity. Striking that balance will require an increased understanding of all the cellular and host factors that control the activation of $\mathrm{T}$ cells.

\section{CONFLICT OF INTEREST DISCLOSURES}

We have read and understood Current Oncology's policy on disclosing conflicts of interest, and we declare the following interests: SDS has participated on an advisory board for Janssen; PSO receives funding from EMD Serono and participates on advisory boards for Myst Therapeutics, Providence, Symphogen, and Tessa Therapeutics.

\section{AUTHOR AFFILIATIONS}

*Princess Margaret Cancer Centre, ${ }^{\dagger}$ Department of Medical Oncology and Hematology, Princess Margaret Cancer Centre, University of Toronto, and 敋epartment of Immunology, University of Toronto, Toronto, ON.

\section{REFERENCES}

1. Wallis CJD, Butaney M, Satkunasivam R, et al. Association of patient sex with efficacy of immune checkpoint inhibitors and overall survival in advanced cancers: a systematic review and meta-analysis. JAMA Oncol 2019;5:529-36.

2. Chen DS, Mellman I. Elements of cancer immunity and the cancer-immune set point. Nature 2017;541:321-30.

3. Steinman RM, Hemmi H. Dendritic cells: translating innate to adaptive immunity. Curr Top Microbiol Immunol 2006;311:17-58.

4. Daley SR, Teh C, Hu DY, Strasser A, Gray DHD. Cell death and thymic tolerance. Immunol Rev 2017;277:9-20.

5. Davis MM. T Cell receptor gene diversity and selection. Annu Rev Biochem 1990;59:475-96.

6. Theofilopoulos AN, Kono DH, Baccala R. The multiple pathways to autoimmunity. Nat Immunol 2017;18:716-24.

7. SchwartzRH.TCell anergy. Annu RevImmunol 2003;21:305-34.

8. Miller JF, Morahan G. Peripheral T cell tolerance. Annu Rev Immunol 1992;10:51-69.

9. Osorio F, Fuentes C, Lopez MN, Salazar-Onfray F, Gonzalez FE. Role of dendritic cells in the induction of lymphocyte tolerance. Front Immunol 2015;6:535.

10. Audiger C, Rahman MJ, Yun TJ, Tarbell KV, Lesage S. The importance of dendritic cells in maintaining immune tolerance. J Immunol 2017;198:2223-31.

11. Schaefer L. Complexity of danger: the diverse nature of damage-associated molecular patterns. JBiol Chem 2014;289: 35237-45.

12. Croft M, Dubey C. Accessory molecule and costimulation requirements for CD4 T cell response. Crit Rev Immunol 2017;37:261-90. 
13. Chen L, Flies DB. Molecular mechanisms of T cell costimulation and co-inhibition. Nat Rev Immunol 2013;13: 227-42.

14. Morales A. BCG: a throwback from the stone age of vaccines opened the path for bladder cancer immunotherapy. Can J Urol 2017;24:8788-93.

15. Sagiv-Barfi I, Czerwinski DK, Levy S, et al. Eradication of spontaneous malignancy by local immunotherapy. Sci Transl Med 2018;10:pii:eaan4488.

16. Sato-Kaneko F, Yao S, Ahmadi A, et al. Combination immunotherapy with TLR agonists and checkpoint inhibitors suppresses head and neck cancer. JCIInsight 2017;2:pii:93397.

17. Iribarren K, Bloy N, Buque A, et al. Trial watch: immunostimulation with toll-like receptor agonists in cancer therapy. Oncoimmunology 2015;5:e1088631.

18. Barber GN. STING. Infection, inflammation and cancer. Nat Rev Immunol 2015;15:760-70.

19. Woo SR, Fuertes MB, Corrales L, et al. STING-dependent cytosolic DNA sensing mediates innate immune recognition of immunogenic tumors. Immunity 2014;41:830-42.

20. Ramanjulu JM, Pesiridis GS, Yang J, et al. Design of amidobenzimidazole STING receptor agonists with systemic activity. Nature 2018;564:439-43.

21. Melero I, Gaudernack G, Gerritsen W, et al. Therapeutic vaccines for cancer: an overview of clinical trials. Nat Rev Clin Oncol 2014;11:509-24.

22. van der Burg SH, Arens R, Ossendorp F, van Hall T, Melief CJ. Vaccines for established cancer: overcoming the challenges posed by immune evasion. Nat Rev Cancer 2016;16:219-33.

23. Rizvi NA, Hellmann MD, Snyder A, et al. Cancer immunology. Mutational landscape determines sensitivity to PD-1 blockade in non-small cell lung cancer. Science 2015;348:124-8.

24. Yarchoan M, Hopkins A, Jaffee EM. Tumor mutational burden and response rate to PD-1 inhibition. $N$ Engl J Med 2017;377:2500-1.

25. Samstein RM, Lee CH, Shoushtari AN, et al. Tumor mutational load predicts survival after immunotherapy across multiple cancer types. Nat Genet 2019;51:202-6.

26. Gubin MM, Zhang X, Schuster H, et al. Checkpoint blockade cancer immunotherapy targets tumour-specific mutant antigens. Nature 2014;515:577-81.

27. Li L, Goedegebuure SP, Gillanders WE. Preclinical and clinical development of neoantigen vaccines. Ann Oncol 2017;28(suppl 12):xii11-17.

28. Keskin DB, Anandappa AJ, Sun J, et al. Neoantigen vaccine generates intratumoral $T$ cell responses in phase IB glioblastoma trial. Nature 2019;565:234-9.

29. Lawler SE, Speranza MC, Cho CF, Chiocca EA. Oncolytic viruses in cancer treatment: a review. JAMA Oncol 2017;3:841-9.

30. Andtbacka RH, Kaufman HL, Collichio F, et al. Talimogene laherparepvec improves durable response rate in patients with advanced melanoma. J Clin Oncol 2015;33:2780-8.

31. Waldmann TA. Cytokines in cancer immunotherapy. Cold Spring Harb Perspect Biol 2018;10:pii:a028472.

32. Floros T, Tarhini AA. Anticancer cytokines: biology and clinical effects of interferon-alpha2, interleukin (IL)-2, IL-15, IL-21, and IL-12. Semin Oncol 2015;42:539-48.

33. Charych DH, Hoch U, Langowski JL, et al. NKTR-214, an engineered cytokine with biased IL2 receptor binding, increased tumor exposure, and marked efficacy in mouse tumor models. Clin Cancer Res 2016;22:680-90.

34. Mayes PA, Hance KW, Hoos A. The promise and challenges of immune agonist antibody development in cancer. Nat Rev Drug Discov 2018;17:509-27.

35. Segal NH, Logan TF, Hodi FS, et al. Results from an integrated safety analysis of urelumab, an agonist anti-CD137 monoclonal antibody. Clin Cancer Res 2017;23:1929-36.
36. Hinrichs CS, Rosenberg SA. Exploiting the curative potential of adoptive T-cell therapy for cancer. Immunol Rev 2014;257:56-71.

37. June CH, O'Connor RS, Kawalekar OU, Ghassemi S, Milone MC. CAR T cell immunotherapy for human cancer. Science 2018;359:1361-5.

38. Robbins PF, Morgan RA, Feldman SA, et al. Tumor regression in patients with metastatic synovial cell sarcoma and melanoma using genetically engineered lymphocytes reactive with NY-ESO-1. J Clin Oncol 2011;29:917-24.

39. D'Angelo SP, Melchiori L, Merchant MS, et al. Antitumour activity associated with prolonged persistence of adoptively transferred NY-ESO-1 ${ }^{\mathrm{C} 259} \mathrm{~T}$ cells in synovial sarcoma. Cancer Discov 2018;8:944-57.

40. Hui E, Cheung J, Zhu J, et al. T Cell costimulatory receptor CD28 is a primary target for PD-1-mediated inhibition. Science 2017;355:1428-33.

41. McLane LM, Abdel-Hakeem MS, Wherry EJ. CD8 T cell exhaustion during chronic viral infection and cancer. Annu Rev Immunol 2019;37:457-95.

42. Crespo J, Sun H, Welling TH, Tian Z, Zou W. T Cell anergy, exhaustion, senescence, and stemness in the tumor microenvironment. Curr Opin Immunol 2013;25:214-21.

43. Zajac AJ, Blattman JN, Murali-Krishna K, et al. Viral immune evasion due to persistence of activated T cells without effector function. J Exp Med 1998;188:2205-13.

44. Gallimore A, Glithero A, Godkin A, et al. Induction and exhaustion of lymphocytic choriomeningitis virus-specific cytotoxic T lymphocytes visualized using soluble tetrameric major histocompatibility complex class I-peptide complexes. J Exp Med 1998;187:1383-93.

45. Barber DL, Wherry EJ, Masopust D, etal. Restoring function in exhausted CD8 T cells during chronic viral infection. Nature 2006;439:682-7.

46. Iwai $\mathrm{Y}$, Ishida M, Tanaka Y, Okazaki T, Honjo T, Minato N. Involvement of PD-L1 on tumor cells in the escape from host immune system and tumor immunotherapy by PD-L1 blockade. Proc Natl Acad Sci U S A 2002;99:12293-7.

47. Wang J, Sanmamed MF, Datar I, et al. Fibrinogen-like protein 1 is a major immune inhibitory ligand of LAG-3. Cell 2019;176:334-347.e312.

48. Esin E. Clinical applications of immunotherapy combination methods and new opportunities for the future. Biomed Res Int 2017;2017:1623679.

49. Sakaguchi S, Sakaguchi N, Asano M, Itoh M, Toda M. Immunologic self-tolerance maintained by activated $\mathrm{T}$ cells expressing IL-2 receptor alpha-chains (CD25). Breakdown of a single mechanism of self-tolerance causes various autoimmune diseases. J Immunol 1995;155:1151-64.

50. Bennett CL, Christie J, Ramsdell F, et al. The immune dysregulation, polyendocrinopathy, enteropathy, $\mathrm{X}$-linked syndrome (IPEX) is caused by mutations of FOXP3. Nat Genet 2001;27:20-1.

51. Wildin RS, Ramsdell F, Peake J, et al. X-Linked neonatal diabetes mellitus, enteropathy and endocrinopathy syndrome is the human equivalent of mouse scurfy. Nat Genet 2001;27:18-20.

52. Brunkow ME, Jeffery EW, Hjerrild KA, et al. Disruption of a new forkhead/winged-helix protein, scurfin, results in the fatal lymphoproliferative disorder of the scurfy mouse. Nat Genet 2001;27:68-73.

53. Josefowicz SZ, Lu LF, Rudensky AY. Regulatory T cells: mechanisms of differentiation and function. Annu Rev Immunol 2012;30:531-64.

54. Shimizu J, Yamazaki S, Sakaguchi S. Induction of tumor immunity by removing CD25+CD4+ T cells: a common basis between tumor immunity and autoimmunity. J Immunol 1999;163:5211-18. 
55. Arce Vargas F, Furness AJS, Solomon I, et al. FC-optimized anti-CD25 depletes tumor-infiltrating regulatory $\mathrm{T}$ cells and synergizes with PD-1 blockade to eradicate established tumors. Immunity 2017;46:577-86.

56. Shang B, Liu Y, Jiang SJ, Liu Y. Prognostic value of tumorinfiltrating FOXP3+ regulatory T cells in cancers: a systematic review and meta-analysis. Sci Rep 2015;5:15179.

57. Simpson TR, Li F, Montalvo-Ortiz W, et al. FC-dependent depletion of tumor-infiltrating regulatory $\mathrm{T}$ cells co-defines the efficacy of anti-CTLA- 4 therapy against melanoma. JExp Med 2013;210:1695-710.

58. Romano E, Kusio-Kobialka M, Foukas PG, et al. Ipilimumabdependent cell-mediated cytotoxicity of regulatory $\mathrm{T}$ cells ex vivo by nonclassical monocytes in melanoma patients. Proc Natl Acad Sci U S A 2015;112:6140-5.

59. Sharma A, Subudhi SK, Blando J, et al. Anti-CTLA-4 immunotherapy does not deplete FOXP3+ regulatory T cells (Tregs) in human cancers. Clin Cancer Res 2019;25:1233-8.

60. De Simone M, Arrigoni A, Rossetti G, et al. Transcriptional landscape of human tissue lymphocytes unveils uniqueness of tumor-infiltrating $\mathrm{T}$ regulatory cells. Immunity 2016;45:1135-47.

61. Plitas G, Konopacki C, Wu K, et al. Regulatory T cells exhibit distinct features in human breast cancer. Immunity 2016;45:1122-34.

62. VillarrealDO, L'Huillier A,ArmingtonS, etal. TargetingCCR8 induces protectiveantitumourimmunityand enhancesvaccineinduced responses in colon cancer. Cancer Res 2018;78:5340-8.

63. Wing JB, Tanaka A, Sakaguchi S. Human FOXP3 ${ }^{+}$regulatory $\mathrm{T}$ cell heterogeneity and function in autoimmunity and cancer. Immunity 2019;50:302-16.

64. Yang M, McKay D, Pollard JW, Lewis CE. Diverse functions of macrophages in different tumor microenvironments. Cancer Res 2018;78:5492-503.

65. Gabrilovich DI. Myeloid-derived suppressor cells. Cancer Immunol Res 2017;5:3-8.
66. Lemos H, Huang L, Prendergast GC, Mellor AL. Immune control by amino acid catabolism during tumorigenesis and therapy. Nat Rev Cancer 2019;19:162-75.

67. Crome SQ, Nguyen LT, Lopez-Verges S, et al. A distinct innate lymphoid cell population regulates tumor-associated T cells. Nat Med 2017;23:368-75.

68. Picard E, Godet Y, Laheurte C, et al. Circulating NKp46 ${ }^{+}$ natural killer cells have a potential regulatory property and predict distinct survival in non-small cell lung cancer. Oncoimmunology 2019;8:e1527498.

69. Poh AR, Ernst M. Targeting macrophages in cancer: from bench to bedside. Front Oncol 2018;8:49.

70. Anani W, Shurin MR. Targeting myeloid-derived suppressor cells in cancer. Adv Exp Med Biol 2017;1036:105-28.

71. Dominguez GA, Condamine T, Mony S, et al. Selective targeting of myeloid-derived suppressor cells in cancer patients using DS-8273a, an agonistic TRAIL-R2 antibody. Clin Cancer Res 2017;23:2942-50.

72. Sivan A, Corrales L, Hubert N, et al. Commensal Bifidobacterium promotes antitumour immunity and facilitates anti-PD-L1 efficacy. Science 2015;350:1084-9.

73. Gopalakrishnan V, Spencer CN, Nezi L, et al. Gut microbiome modulates response to anti-PD-1 immunotherapy in melanoma patients. Science 2018;359:97-103.

74. Routy B, Le Chatelier E, Derosa L, et al. Gut microbiome influences efficacy of PD-1-based immunotherapy against epithelial tumors. Science 2018;359:91-7.

75. Levy M, Thaiss CA, Elinav E. Metabolites: messengers between the microbiota and the immune system. Genes Dev 2016;30:1589-97.

76. Balmer ML, Ma EH, Bantug GR, et al. Memory $\mathrm{CD}^{+} \mathrm{T}$ cells require increased concentrations of acetate induced by stress for optimal function. Immunity 2016;44:1312-24.

77. Luu M, Weigand K, Wedi $\mathrm{F}$, et al. Regulation of the effector function of $\mathrm{CD}^{+} \mathrm{T}$ cells by gut microbiota-derived metabolite butyrate. Sci Rep 2018;8:14430. 